\title{
Derived Sets in Multiobjective Optimization
}

\author{
W. W. Breckner
}

\begin{abstract}
In the present paper the theory of derived sets established by $M$. R. Hestenes and J. W. Nieuwenhuis for optimization problems with a real-valued objective function and a finite number of constraints is extended to multiobjective optimization problems. The main result asserts that local solutions of weak multiobjective optimization problems satisfy a multiplier rule.
\end{abstract}

Keywords: Multiobjective optimization, necessary optimality conditions, derived set

AMS subject classiflcation: $90 \mathrm{C} 29,49 \mathrm{~K} 27$

\section{Introduction}

Consider the following optimization problem:

$\left(\mathbf{P}_{1}\right)$ Maximize $f_{1}(x)$ subject to $x \in X, f_{2}(x) \geq 0, f_{3}(x)=0$

where $X$ is a non-empty set and $f_{1}: X \rightarrow R, f_{2}: X \rightarrow R^{m_{2}}, f_{3}: X \rightarrow R^{m_{3}}$. The notation $f_{2}(x) \geq 0$ means that each component of $f_{2}(x)$ is non-negative.

Let $m=1+m_{2}+m_{3}$. By making use of so-called derived sets in the image space $R^{m}$ of the vector function $f=\left(f_{1}, f_{2}, f_{3}\right): X \rightarrow R^{m}$, Hestenes has shown in [8 - 10] that the solutions of problem $\left(P_{1}\right)$ satisfy a certain multiplier rule which generalizes the classical Lagrange multiplier rule concerning the optimization of real-valued functions subject to equality constraints. Hestenes' multiplier rule played an important role in optaining necessary optimality conditions in optimization theory, variational theory and control theory. It also has been one of the starting points for the present paper.

According to the definition given in [10: p. 368], a non-empty subset $D \subseteq R^{m}$ is said to be a derived set for the function $f$ at the point $x_{0} \in X$ if for every $n \in N$ and every $n$-tuple $\left(d^{1}, \ldots, d^{n}\right)$ of points belonging to $D$ there exist a number $r>0$ and a function $\omega:[0, r]^{n} \rightarrow X$ such that the following conditions are satisfied:

(i) $\lim _{t \rightarrow 0} \frac{1}{\|\pi\|}\left(f(\omega(t))-f\left(x_{0}\right)-A(t)\right)=0$, where $A(t)=t_{1} d^{1}+\ldots+t_{n} d^{n}$ for all $t=\left(t_{1}, \ldots, t_{n}\right) \in R^{n}$.

(ii) $\dot{\omega}(0)=x_{0}$.

(iii) $f \circ w$ (the composite function) is continuous.

W. W. Breckner: Univ. Babeş-Bolyai, Fac. de Matematică, Str. Kogălniceanu 1, RO - 3400 Cluj-Napoca, Romania 
A convex cone in $R^{m}$ that is a derived set for $f$ at $x_{0} \in X$ is said to be a derived convex cone for $f$ at $x_{0}$.

The above-mentioned multiplier rule by.Hestenes (see [10: Theorem 10.1]) asserts that, if $x_{0} \in X$ is a solution to problem $\left(\mathrm{P}_{1}\right)$ and $D \subseteq R \times R^{m_{2}} \times R^{m_{3}}$ is a derived set for $f$ at $x_{0}$, then there exists a vector

$$
\left(\lambda_{1}^{*}, \lambda_{2}^{*}, \lambda_{3}^{*}\right) \in R \times R^{m_{2}} \times R^{m_{3}} \backslash\{(0,0,0)\}
$$

such that

$$
\begin{gathered}
\lambda_{1}^{*} \geq 0 \quad \text { and } \quad \lambda_{2}^{*} \geq 0 \\
\sup \left\{d_{1} \lambda_{1}^{*}+\left\langle d_{2}, \lambda_{2}^{*}\right\rangle+\left\langle d_{3}, \lambda_{3}^{*}\right\rangle \mid\left(d_{1}, d_{2}, d_{3}\right) \in D\right\} \leq 0 \\
\left\langle f_{2}\left(x_{0}\right), \lambda_{2}^{*}\right\rangle=0 .
\end{gathered}
$$

Nieuwenhuis [14] has investigated a generalization of problem $\left(P_{1}\right)$, namely the following problem:

$\left(\mathbf{P}_{2}\right) \quad$ Maximize $f_{1}(x)$ subject to $x \in X, f_{2}(x) \in K_{2}, f_{3}(x) \in K_{3}$

where $K_{2}$ is a closed convex cone in the space $R^{m_{2}}$ with int $K_{2} \neq \emptyset$, while $K_{3}$ is a closed convex cone in the space $R^{m_{3}}$. Associating with this problem a suitable concept of a derived set, he succeeded to prove a multiplier rule (see [14: Theorem 3.1]) for the solutions of problem $\left(\mathrm{P}_{2}\right)$. But, in contrast to Hestenes' multiplier rule, in this new multiplier rule an orthogonality condition of type (1.3) is missing.

In the present paper we carry on Hestenes' and Nieuwenhuis' researches concerning derived sets. Three substantial improvements will be achieved. Firstly, we introduce derived sets for multiobjective optimization problems, i.e. problems in which it is required to maximize a vector function $f_{1}: X \rightarrow R^{m_{1}}$ on a non-empty subset $X$ of a topological space subject to constraints of the form $f_{2}(x) \in K_{2}$ and $f_{3}(x) \in K_{3}$, where $f_{2}, f_{3}, K_{2}$ and $K_{3}$ have the same meaning as in problem $\left(\mathrm{P}_{2}\right)$. Secondly, in our concept of a derived set the functions $f_{1}, f_{2}$ and $f_{3}$, occurring in the formulation of the optimization problem, cease to play the same role. This manifests itself through the fact that the conditions imposed on $f_{1}$ and $f_{2}$ are weaker than those imposed on $f_{3}$. Thirdly, by using this generalized concept of a derived set we state a multiplier rule which holds even for local solutions of weak multiobjective optimization problems and in which an orthogonality condition of type (1.3) also occurs. All these improvements of the theories known till now are obtained due to a subtle modification of the ideas applied by Nieuwenhuis in his research. Nevertheless a sophistication of the proofs was inevitable.

So far as we know Hestenes' image space technique for deriving necessary optimality conditions has not been used in multiobjective optimization hitherto. However, multiplier rules for diverse multiobjective optimization problems (especially for Pareto optimization problems) have already been obtained by other methods (see, e.g., [7, 11 $-13,15-18])$.

The image space technique is very useful in optimization, because it merely requires that the space in which the solutions of the optimization problem are sought is a topological one. This assumption can always be satisfied. But not any of algebraical structure of the underlying space is needed. 
Finally, it should be mentioned that for ordinary (scalar) optimization problems not only the above-mentioned extension of Hestenes' concept of a derived set owing to Nieuwenhuis is known. There also exist other enlargements (see [2] and [4]). For some interesting applications of the derived sets the reader is referred to $[1,5,6,8,9,20]$.

\section{Notations and preliminaries}

Throughout this paper, $N$ is the set of all positive integers, $R$ is the set of all real numbers, and $R^{m}$ is, for every $m \in N$, the usual $m$-dimensional Euclidean space of all $m$-tuples $x=\left(x_{1}, \ldots, x_{m}\right)$ of real numbers. The inner product of two vectors $x, y \in R^{m}$ is denoted by $\langle x, y\rangle$. If $x \in R^{m}$, then $\|x\|$ marks its Euclidean norm.

The subset of $R^{m}$, consisting of all vectors $x=\left(x_{1}, \ldots, x_{m}\right)$ with $x_{j} \geq 0$ for each $j \in\{1, \ldots, m\}$, is denoted by $R_{+}^{m}$. In particular, $R_{+}$designates the set of all nonnegative real numbers. Given any number $r>0$, we put

$$
B_{+}^{m}(r)=\left\{x \in R_{+}^{m} \mid\|x\| \leq r\right\}
$$

If $M$ is a subset of the space $R^{m}$, then we denote by int $M$ its interior. A subset $K$ of the space $R^{m}$ is said to be a:

i) cone if it is not empty and if $a x \in K$ whenever $a \in R_{+}$and $x \in K$

ii) convex cone if it is both a convex set and a cone.

If $M$ is a non-empty subset of the space $R^{m}$, then the sets

$$
\mathcal{K}(M)=\left\{y \in R^{m} \mid y=a_{1} x^{1}+\ldots+a_{k} x^{k} \quad\left(k \in N, a \in R_{+}^{k}, x^{1}, \ldots, x^{k} \in M\right)\right\}
$$

and

$$
M^{*}=\left\{y \in R^{m} \mid\langle x, y\rangle \geq 0 \text { for all } x \in M\right\}
$$

are convex cones. They are called the convex cone generated by $M$ and the dual cone of $M$, respectively. It is well-known (see, for instance, [3: Satz 2.42]) that $M$ is a closed convex cone if and only if $\left(M^{*}\right)^{*}=M$.

In our investigations we shall use the following two results referring to convex cones.

Proposition 2.1. Let $K$ and $L$ be convex cones in the space $R^{m}$, of which $L$ is closed. Then $K^{*} \cap L \neq\{0\}$ if and only if the condition

$$
\left\{x^{j} \mid j=1, \ldots, m+1\right\}^{*} \cap L \neq\{0\} \quad \text { whenever } x^{1}, \ldots, x^{m+1} \in K
$$

is satisfied.

Proposition 2.2. Let $t^{0}$ be a vector in the space $R^{m}$, let $K$ be a closed convex cone in the space $R^{p}$, and let $F: R^{m} \rightarrow R^{p}$ be a function satisfying the following conditions:

(i) $F$ is continuous

(ii) $F$ is differentiable at 0 
(iii) $F(0) \in K, F^{\prime}(0)\left(t^{0}\right) \in K$ and $F^{\prime}(0)\left(R^{m}\right)+K=R^{p}$.

Then there exist a sequence $\left(a_{n}\right)$ of positive numbers and a sequence $\left(t^{n}\right)$ of vectors in the space $R^{m}$ such that

$$
\lim _{n \rightarrow \infty} a_{n}=0, \quad \lim _{n \rightarrow \infty} t^{n}=t^{0}, \quad F\left(a_{n} t^{n}\right) \in K \quad \text { for all } n \in N .
$$

The first of these two propositions has been stated in [14: Lemma 3.1], while the last one is a special case of [19: Theorem 1].

\section{The weak multiobjective optimization problem and $K$-derived sets}

Let $m_{1}, m_{2}$ and $m_{3}$ be positive integers and $m=m_{1}+m_{2}+m_{3}$. In what follows the corresponding space $R^{m}$ will always be conceived as the product space $R^{m_{1}} \times R^{m_{2}} \times R^{m_{3}}$, i.e. any vector $y \in R^{m}$ is identified with a certain triple $\left(y_{1}, y_{2}, y_{3}\right) \in R^{m_{1}} \times R^{m_{2}} \times R^{m_{3}}$. In particular, the zero-vector in $R^{m}$ is $0=\left(0_{1}, 0_{2}, 0_{3}\right)$, where $0_{i}(i \in\{1,2,3\})$ is the zero-vector in $R^{m_{i}}$.

In accord with the above made convention concerning $R^{m}$, any function $\phi$ from a non-empty set $M$ to $R^{m}$ will be interpreted as a triple $\left(\phi_{1}, \phi_{2}, \phi_{3}\right)$, where $\phi_{i}: M \rightarrow R^{m_{i}}$ $(i \in\{1,2,3\})$ are functions such that

$$
\phi(x)=\left(\phi_{1}(x), \phi_{2}(x), \phi_{3}(x)\right) \text { for all } x \in M
$$

Let $X$ be a non-empty subset of a topological space $\mathcal{X}$, and let $f: X \rightarrow R^{m}$. Further, let $K_{1}, K_{2}$ and $K_{3}$ be convex cones in the spaces $R^{m_{1}}, R^{m_{2}}$ and $R^{m_{3}}$, respectively, satisfying the following assumptions:

i) int $K_{1} \neq \emptyset$ and int $K_{2} \neq \emptyset$

ii) $K_{2}$ and $K_{3}$ are closed.

Obviously, $K=K_{1} \times K_{2} \times K_{3}$ is a convex cone in the space $R^{m}$.

Set

$$
S=\left\{x \in X \mid f_{2}(x) \in K_{2} \text { and } f_{3}(x) \in K_{3}\right\} .
$$

A point $x_{0} \in \mathcal{X}$ is said to be a

i) weakly $K_{1}$-maximal point of $f_{1}$ over $S$ if $x_{0} \in S$ and $\left(f_{1}\left(x_{0}\right)+\operatorname{int} K_{1}\right) \cap f_{1}(S)=\emptyset$;

ii) local weakly $K_{1}$-maximal point of $f_{1}$ over $S$ if $x_{0} \in S$ and if there is a neighbourhood $V$ of $x_{0}$ such that $\left(f_{1}\left(x_{0}\right)+\right.$ int $\left.K_{1}\right) \cap f_{1}(S \cap V)=0$.

The problem of finding the weakly $K_{1}$-maximal points of $f_{1}$ over $S$ is called a weak multiobjective optimization problem and shortly expressed as

(WMOP) $\quad\left\{f_{1}(x) \mid x \in X, f_{2}(x) \in K_{2}, f_{3}(x) \in K_{3}\right\} \longrightarrow K_{1}$ max weakly.

The introduction of problem (WMOP) allows to call the weakly $K_{1}$-maximal points of $f_{1}$ over $S$ solutions to problem (WMOP). By analogy, the local weakly $K_{1}$-maximal points of $f_{1}$ over $S$ can be named local solutions to problem (WMOP). 
It should be noted that the problem $\left(P_{2}\right)$, formulated in Section 1 , is a special case of the problem (WMOP). Indeed, to see this we choose $m_{1}=1$ and $K_{1}=R_{+}$, on the one hand, and endow the non-empty set $X$ occurring in problem $\left(\mathrm{P}_{2}\right)$ with the indiscrete topology, on the other hand.

The main notion we shall use for obtaining a necessary optimality condition for the local solutions to problem (WMOP) is that of a $K$-derived convex cone. In order to introduce this concept let $x_{0}$ be any point in $X$.

An $n$-tuple $\left(d^{1}, \ldots, d^{n}\right)$ of points of the space $R^{m}$ is said to be a $K$-gradient of $f$ at $x_{0}$ if there exist a number $r>0$, a function $\omega: B_{+}^{n}(r) \rightarrow X$ and a function $\rho: B_{+}^{n}(r) \rightarrow R^{m}$ such that the following conditions are satisfied:

(A) $f(\omega(t))-f\left(x_{0}\right)-\left(t_{1} d^{1}+\ldots+t_{n} d^{n}\right)-\|t\| \rho(t) \in K$ for all $t=\left(t_{1}, \ldots, t_{n}\right) \in$ $B_{+}^{n}(r)$.

(B) $\omega(0)=x_{0}$ and $\omega$ is continuous at 0 .

$\left(C_{1}\right)$ There exists a point $y_{1}^{0} \in K_{1}$ so that, for each number $\varepsilon>0$, there is a number $r_{\varepsilon} \in(0, r)$ such that

$$
\rho_{1}(t)+\varepsilon y_{1}^{0} \in K_{1} \quad \text { whenever } t \in B_{+}^{n}\left(r_{e}\right)
$$

$\left(\mathrm{C}_{2}\right)$ There exists a point $y_{2}^{0} \in K_{2}$ so that, for each number $\varepsilon>0$, there is a number $r_{e} \in(0, r]$ such that

$$
\rho_{2}(t)+\varepsilon y_{2}^{0} \in K_{2} \quad \text { whenever } t \in B_{+}^{n}\left(r_{e}\right)
$$

$\left(\mathrm{C}_{3}\right) \rho_{3}(0)=0_{3}$ and $\rho_{3}$ is continuous.

A subset $D \subseteq R^{m}$ is said to be a

i) $K$-derived set for $f$ at $x_{0}$ if it is not empty and if every $m+1$-tuple of points belonging to $D$ is a $K$-gradient of $f$ at $x_{0}$;

ii) $K$-derived convex cone for $f$ at $x_{0}$ if it is both a $K$-derived set for $f$ at $x_{0}$ and a convex cone.

Remark 3.1. If $\rho_{1}(0)=0_{1}$. and $\rho_{1}$ is continuous at 0 , then condition $\left(C_{1}\right)$ is satisfied. Indeed, let $y_{1}^{0}$ be any interior point of $K_{1}$. Then $K_{1}-\varepsilon y_{1}^{0}$ is for each number $\varepsilon>0$ a neighbourhood of $0_{1}$. In consequence, in view of the assumptions on $\rho_{1}$ there must exist for each number $\varepsilon>0$ a number $r_{\varepsilon} \in(0, r]$ such that inclusion (3.1) holds.

Of course a similar remark can be made concerning $\rho_{2}$ and condition $\left(\mathrm{C}_{2}\right)$.

Remark 3.2. The functions $\rho_{1}, \rho_{2}$ and $\rho_{3}$ that occur in our definition of the $K$ gradient of $f$ are linked with the functions $r_{0}, r_{y}$ and $r_{z}$ used in [14]. An attentive analysis reveals that the proof of Theorem 3.1 in [14] employs the continuity of $r_{z}$ on $R_{+}^{p+2}$, although this property has not been supposed. If we add this assumption, then it follows by Remark 3.1 that the definition of a derived convex cone suggested by Lemma 3.2 from [14] is a special case of our concept of a $K$-derived convex cone. 
Proposition 3.1. Let $x_{0} \in X$, and let $M$ be a non-empty subset of the space $R^{m}$ such that, for all $n \in N$ and all $y^{1}, \ldots, y^{n} \in M$, the $n$-tuple $\left(y^{1}, \ldots, y^{n}\right)$ is a $K$-gradient of $f$ at $x_{0}$. Then $\mathcal{K}(M)$ is a $K$-derived convex cone for $f$ at $x_{0}$.

Proof. Let $d^{1}, \ldots, d^{m+1}$ be vectors in $\mathcal{K}(M)$. Then we can select a finite number of vectors $y^{1}, \ldots, y^{n} \in M$ as well as numbers $a_{j k} \in R_{+}(j \in\{1, \ldots, m+1\}$ and $k \in$ $\{1, \ldots, n\})$ such that

$$
d^{j}=\sum_{k=1}^{n} a_{j k} y^{k} \text { for all } j \in\{1, \ldots, m+1\}
$$

Since $\left(y^{1}, \ldots, y^{n}\right)$ is a $K$-gradient of $f$ at $x_{0}$, there exist a number $r>0$, a function $\omega: B_{+}^{n}(r) \rightarrow X$ and a function $\rho: B_{+}^{n}(r) \rightarrow R^{m}$ satisfying the condition

$$
f(\omega(t))-f\left(x_{0}\right)-\left(t_{1} y^{1}+\ldots+t_{n} y^{n}\right)-\|t\| \rho(t) \in K
$$

for all $t=\left(t_{1}, \ldots, t_{n}\right) \in B_{+}^{n}(r)$ as well as the conditions (B), $\left(\mathrm{C}_{1}\right)-\left(\mathrm{C}_{3}\right)$.

Let $A=\left(A_{1}, \ldots, A_{n}\right): R^{m+1} \rightarrow R^{n}$ be the mapping, whose components

$$
A_{k}: R^{m+1} \rightarrow R \quad(k \in\{1, \ldots, n\})
$$

are defined by

$$
A_{k}(u)=\sum_{j=1}^{m+1} u_{j} a_{j k} \text { for all } u=\left(u_{1}, \ldots, u_{m+1}\right) \in R^{m+1}
$$

For all $u=\left(u_{1}, \ldots, u_{m+1}\right) \in R_{+}^{m+1}$ we have

$$
\begin{aligned}
& u_{1} d^{1}+\ldots+u_{m+1} d^{m+1}=A_{1}(u) y^{1}+\ldots+A_{n}(u) y^{n} \\
& A(u) \in R_{+}^{n} \quad \text { and } \quad\|A(u)\| \leq \sum_{k=1}^{n} A_{k}(u) \leq a\|u\|
\end{aligned}
$$

where

$$
a=1+\sum_{j=1}^{m+1} \sum_{k=1}^{n} a_{j k} .
$$

Set $\bar{r} \doteq r / a$. In view of (3.4) it follows that $A$ maps $B_{+}^{m+1}(\bar{r})$ into $B_{+}^{n}(r)$. Consequently we can define the functions

$$
\bar{\omega}: B_{+}^{m+1}(\bar{r}) \rightarrow X \quad \text { and } \quad \bar{\rho}: B_{+}^{m+1}(\bar{r}) \rightarrow R^{m}
$$

by

$$
\bar{\omega}(u)=\omega(A(u)) \quad \text { and } \quad \bar{\rho}(u)= \begin{cases}\|A(u)\| /\|u\| \rho(A(u)) & \text { if } u \neq 0 \\ 0 & \text { if } u=0,\end{cases}
$$

respectively. We claim that for $\bar{r}, \bar{\omega}$ and $\bar{\rho}$ the following properties are valid: 
(六) $f(\bar{\omega}(u))-f\left(x_{0}\right)-\left(u_{1} d^{1}+\ldots+u_{m+1} d^{m+1}\right)-\|u\| \bar{\rho}(u) \in K$ for all elements $u=\left(u_{1}, \ldots, u_{m+1}\right) \in B_{+}^{m+1}(\bar{r})$.

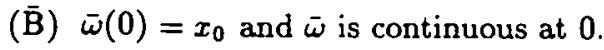

$\left(\bar{C}_{1}\right)$ For each number $\varepsilon>0$ there is a number $\bar{r}_{\varepsilon} \in(0, \bar{r}]$ such that

$$
\bar{\rho}_{1}(u)+\varepsilon y_{1}^{0} \in K_{1} \quad \text { whenever } u \in B_{+}^{m+1}\left(\bar{r}_{\varepsilon}\right) \text {. }
$$

$\left(\overline{\mathrm{C}}_{2}\right)$ For each number $\varepsilon>0$ there is a number $\bar{r}_{e} \in(0, \bar{r}]$ such that

$$
\bar{\rho}_{2}(u)+\varepsilon y_{2}^{0} \in K_{2} \quad \text { whenever } u \in B_{+}^{m+1}\left(\bar{r}_{e}\right) \text {. }
$$

$\left(\overline{\mathrm{C}}_{3}\right) \bar{\rho}_{3}(0)=0_{3}$ and $\bar{\rho}_{3}$ is continuous.

Indeed, from (3.2) and (3.3) it follows that property $(\bar{A})$ holds. Taking into account that $A(0)=0$ and that $A$ is continuous at 0 , we see that condition (B) implies property $(\overline{\mathrm{B}})$. Next we prove property $\left(\overline{\mathrm{C}}_{1}\right)$. Let $\varepsilon>0$ be given. In view of condition $\left(\mathrm{C}_{1}\right)$ there exists a number $r_{1} \in(0, r]$ such that

$$
\rho_{1}(t)+\frac{\varepsilon}{a} y_{1}^{0} \in K_{1} \quad \text { whenever } t \in B_{+}^{n}\left(r_{1}\right) .
$$

Choose $\bar{r}_{e}=r_{1} / a$. By (3.4) we have $A(u) \in B_{+}^{n}\left(r_{1}\right)$ for all $u \in B_{+}^{m+1}\left(\bar{r}_{e}\right)$, and hence (3.6) yields

$$
a \rho_{1}(A(u)) \in K_{1}-\varepsilon y_{1}^{0} \quad \text { whenever } u \in B_{+}^{m+1}\left(\bar{r}_{\varepsilon}\right) .
$$

Taking into account that $K_{1}-\varepsilon y_{1}^{0}$ is a convex set containing $0_{1}$ and that

$$
0 \leq \frac{\|A(u)\|}{a\|u\|} \leq 1 \text { for all } u \in R_{+}^{m+1} \backslash\{0\} \text {, }
$$

it follows from (3.7) that (3.5) is true. A similar proof shows that property $\left(\overline{\mathrm{C}}_{2}\right)$ is also true. Finally, observe that condition $\left(\mathrm{C}_{3}\right)$, equality $A(0)=0$ and the continuity of $A$ imply property $\left(\overline{\mathrm{C}}_{3}\right)$. Consequently, all the properties $(\overline{\mathrm{A}}),(\overline{\mathrm{B}}),\left(\overline{\mathrm{C}}_{1}\right)-\left(\overline{\mathrm{C}}_{3}\right)$ are true as claimed.

These properties show that $\left(d^{1}, \ldots, d^{m+1}\right)$ is a $K$-gradient of $f$ at $x_{0}$. Since the vectors $d^{1}, \ldots, d^{m+1}$ were arbitrarily chosen in $\mathcal{K}(M)$, it follows that $\mathcal{K}(M)$ is a $K$ derived set for $f$ at $x_{0}$. On the other hand, as it has already been remarked in Section 2 , the set $\mathcal{K}(M)$ is a convex cone. Consequently $\mathcal{K}(M)$ is a $K$-derived convex cone for $f$ at $x_{0}$

Corollary 3.2. Let $x_{0}$ be a point in $X$, and let $M$ be a non-empty subset of the space $R^{m}$ such that, for every $n \in N$ and every $n$-tuple $\left(y^{1}, \ldots, y^{n}\right)$ of points belonging to $M$, there exist a number $r>0$ and a function $\omega: B_{+}^{n}(r) \rightarrow X$ satisfying the following conditions:

(i) $\lim _{t \rightarrow 0} \frac{1}{\|t\|}\left(f(\omega(t))-f\left(x_{0}\right)-A(t)\right)=0$, where $A(t)=t_{1} y^{1}+\ldots+t_{n} y^{n}$ for all $t=\left(t_{1}, \ldots, t_{n}\right) \in R^{n}$. 
(ii) $\omega(0)=x_{0}$ and $\omega$ is continuous at 0 .

(iii) $f \circ \omega$ (the composite function) is continuous.

Then $\mathcal{K}(M)$ is a $K$-derived convex cone for $f$ at $x_{0}$.

Proof. Let $n$ be any positive integer, and let $y^{1}, \ldots, y^{n} \in M$. Then there exist a number $r>0$ and a function $\omega: B_{+}^{n}(r) \rightarrow X$ such that the conditions (i) - (iii) are satisfied. Define $\rho: B_{+}^{n}(r) \rightarrow R^{m}$ by

$$
\rho(t)= \begin{cases}\frac{1}{\|t\|}\left(f(\omega(t))-f\left(x_{0}\right)-A(t)\right) & \text { if } t \neq 0 \\ 0 & \text { if } t=0 .\end{cases}
$$

Then we have

$$
f(\omega(t))-f\left(x_{0}\right)-A(t)-\|t\| \rho(t)=0 \text { for all } t \in B_{+}^{n}(r)
$$

and hence

$$
f(\omega(t))-f\left(x_{0}\right)-A(t)-\|t\| \rho(t) \in K \quad \text { for all } t \in B_{+}^{n}(r) .
$$

On the other hand, it follows from conditions (i) and (iii) that $\rho$ is continuous. Together with Remark 3.1 this implies that the conditions $\left(C_{1}\right)-\left(C_{3}\right)$ are satisfied.

Summing up, the number $r$ and the functions $\omega$ and $\rho$ satisfy (3.8), (ii), $\left(\mathrm{C}_{1}\right)-\left(\mathrm{C}_{3}\right)$. Consequently $\left(y^{1}, \ldots, y^{n}\right)$ is a $K$-gradient of $f$ at $x_{0}$. But $n \in N$ and $y^{1}, \ldots, y^{n} \in M$ were arbitrarily chosen. Thus Proposition 3.1 is applicable and yields that $\mathcal{K}(M)$ is a $K$-derived convex cone for $f$ at $x_{0}$

Corollary 3.2 points out that the concept of a $K$-derived convex cone introduced in this paper is a generalization of Hestenes' concept of a derived convex cone, which was recalled in Section 1.

\section{The multiplier rule}

Now we are in a position to state a multiplier rule for the local solutions of problem (WMOP): For this end all assumptions and notations specified in the preceding section will be kept on.

Theorem 4.1. Let $x_{0} \in \mathcal{X}$ be a local solution to problem (WMOP) and let $D \subseteq R^{m}$ be a $K$-derived convex cone for $f$ at $x_{0}$. Then there exists a vector

$$
\left(\lambda_{1}^{*}, \lambda_{2}^{*}, \lambda_{3}^{*}\right) \in K_{1}^{*} \times K_{2}^{*} \times K_{3}^{*} \backslash\left\{\left(0_{1}, 0_{2}, 0_{3}\right)\right\}
$$

such that

$$
\sup \left\{\left\langle d_{1}, \lambda_{1}^{*}\right\rangle+\left\langle d_{2}, \lambda_{2}^{*}\right\rangle+\left\langle d_{3}, \lambda_{3}^{*}\right\rangle \mid\left(d_{1}, d_{2}, d_{3}\right) \in D\right\} \leq 0
$$

and .

$$
\left\langle f_{2}\left(x_{0}\right), \lambda_{2}^{*}\right\rangle=0
$$


Proof. Let $d^{1}, \ldots, d^{m+1}$ be vectors belonging to $D$. Define the mappimg $A$ : $R^{m+1} \rightarrow R^{m}$ by

$$
A(t)=t_{1} d^{1}+\ldots+t_{m+1} d^{m+1} \text { for all } t=\left(t_{1}, \ldots, t_{m+1}\right) \in R^{m+1} .
$$

Next introduce the sets

$$
L=\left\{u \in K_{2}^{*} \mid\left\langle f_{2}\left(x_{0}\right), u\right\rangle=0\right\}
$$

and

$$
M=\left\{y \in R^{m} \mid A(t) \in y+K_{1} \times L^{*} \times K_{3} \text { for some } t \in \operatorname{int} R_{+}^{m+1}\right\} .
$$

We claim that the origin of the space $R^{m}$ is not an interior point of $M$. To prove this assertion, we assume that the origin $\left(0_{1}, 0_{2}, 0_{3}\right)$ of the space $R^{m}$ is an interior point of $M$. Then there exists a balanced neighbourhood $V_{3}$ of $0_{3}$ such that $\left\{0_{1}\right\} \times\left\{0_{2}\right\} \times V_{3} \subseteq M$. In consequence there exists for each $y_{3} \in V_{3}$ a point $t \in$ int $R_{+}^{m+1}$ such that $A_{3}(t) \in y_{3}+K_{3}$. Thus we have $V_{3} \subseteq A_{3}\left(R^{m+1}\right)+K_{3}$. This relation implies that

$$
R^{m_{3}}=A_{3}\left(R^{m+1}\right)+K_{3} .
$$

Since $L^{*} \supseteq\left(K_{2}^{*}\right)^{*}=K_{2}$, it results that int $L^{*} \neq 0$. Pick out a point

$$
z=\left(z_{1}, z_{2}, z_{3}\right) \in \text { int } K_{1} \times \text { int } L^{*} \times K_{3} \text {. }
$$

Inasmuch as $M$ is a neighbourhood of the origin of the space $R^{m}$, there exists a number $a>0$ such that $a z \in M$. Hence we can find an interior point $t^{0}$ of $R_{+}^{m+1}$ for which

$$
\begin{aligned}
& A_{1}\left(t^{0}\right) \in a z_{1}+K_{1} \subseteq \text { int } K_{1}+K_{1} \subseteq \text { int } K_{1} \\
& A_{2}\left(t^{0}\right) \in a z_{2}+L^{*} \subseteq \text { int } L^{*}+L^{*} \subseteq \text { int } L^{*} \\
& A_{3}\left(t^{0}\right) \in a z_{3}+K_{3} \subseteq K_{3}+K_{3} \subseteq K_{3} .
\end{aligned}
$$

We now take into consideration that $\left(d^{1}, \ldots, d^{m+1}\right)$ is a $K$-gradient of $f$ at $x_{0}$. So we can find a number $r>0$, a function $\omega: B_{+}^{m+1}(r) \rightarrow X$ and a function $\rho: B_{+}^{m+1}(r) \rightarrow R^{m}$ that satisfy the following conditions:

(a) $f(\omega(t))-f\left(x_{0}\right)-A(t)-\|t\| \rho(t) \in K$ for all $t \in B_{+}^{m+1}(r)$.

(b) $\omega(0)=\dot{x}_{0}$ and $\omega$ is continuous at 0 .

(c) There exists a point $y_{1}^{0} \in K_{1}$ so that for each number $\varepsilon>0$ there is a number $r_{e} \in(0, r]$ such that $\rho_{1}(t)+\varepsilon y_{1}^{0} \in K_{1}$ whenever $t \in B_{+}^{m+1}\left(r_{e}\right)$.

(c) There exists a point $y_{2}^{0} \in K_{2}$ so that for each number $\varepsilon>0$ there is a number $r_{\varepsilon} \in(0, r]$ such that $\rho_{2}(t)+\varepsilon y_{2}^{0} \in K_{2}$ whenever $t \in B_{+}^{m+1}\left(r_{\varepsilon}\right)$.

(c) $\rho_{3}(0)=0_{3}$ and $\rho_{3}$ is continuous.

Let $\bar{\rho}_{3}: R^{m+1} \rightarrow R^{m_{3}}$ be a continuous extension of $\rho_{3}$. Define $F: R^{m+1} \rightarrow R^{m a}$ by

$$
F(t)=f_{3}\left(x_{0}\right)+A_{3}(t)+\|t\| \bar{\rho}_{3}(t) .
$$


This function is continuous. In view of condition $\left(c_{3}\right)$ it is differentiable at 0 . Moreover, in virtue of $F^{\prime}(0)=A_{3}$, we have

$$
F^{\prime}(0)\left(t^{0}\right) \in K_{3} \quad \text { and } \quad F^{\prime}(0)\left(R^{m+1}\right)+K_{3}=R^{m_{3}} .
$$

In addition to these properties of $F$ we have $F(0)=f_{3}\left(x_{0}\right) \in K_{3}$. Summing up, all the hypotheses of Proposition 2.2 are satisfied by $t^{0}, K_{3}$ and $F$. By applying Proposition 2.2 we conclude that there exist a sequence $\left(a_{n}\right)$ of positive numbers and a sequence $\left(t^{n}\right)$ of vectors in the space $R^{m+1}$ such that

$$
\lim _{n \rightarrow \infty} a_{n}=0 \quad \text { and } \quad \lim _{n \rightarrow \infty} t^{n}=t^{0}
$$

and

$$
F\left(a_{n} t^{n}\right) \in K_{3} \quad \text { for all } n \in N .
$$

Since the sequence $\left(t^{n}\right)$ converges to an interior point of the set $R_{+}^{m+1}$ and the sequence $\left(a_{n} t^{n}\right)$ converges to the origin of the space $R^{m+1}$, we can assume without loss of the generality that all the terms $a_{n} t^{n}(n \in N)$ lie in $B_{+}^{m+1}(r)$. Then all the points $\omega\left(a_{n} t^{n}\right)(n \in N)$ lie in $X$. Moreover, for sufficiently large $n$, these points lie even in $S$.

To see this, we firstly remark that (a) and (4.4) imply

$$
f_{3}\left(\omega\left(a_{n} t^{n}\right)\right) \in K_{3}+F\left(a_{n} t^{n}\right) \subseteq K_{3}+K_{3} \subseteq K_{3} \text { for all } n \in N \text {. }
$$

Next we define the function $G: B_{+}^{m+1}(r) \rightarrow R^{m_{2}}$ by

$$
G(t)=f_{2}\left(x_{0}\right)+A_{2}(t)+\|t\| \rho_{2}(t) .
$$

For this function we can find a number $p_{0} \in N$ such that

$$
\left\langle G\left(a_{n} t^{n}\right), u\right\rangle>0 \text { for all } p_{0} \leq n \in N
$$

and all $u \in U$, where $U=\left\{u \in K_{2}^{*} \mid\|u\|=1\right\}$. Indeed, if we suppose the contrary, there exist a subsequence $\left(a_{n_{k}} t^{n_{k}}\right)$ of the sequence $\left(a_{n} t^{n}\right)$ and a sequence $\left(u^{k}\right)$ of points belonging to the compact set $U$ such that

$$
\left\langle G\left(a_{n_{k}} t^{n_{k}}\right), u^{k}\right\rangle \leq 0 \text { for all } k \in N
$$

Without loss of the generality we can assume that the sequence $\left(u^{k}\right)$ converges to a point $u^{0} \in U$. Passing to the limit in (4.7) when $k \rightarrow \infty$, we obtain $\left\langle G(0), u^{0}\right\rangle \leq 0$. In other words, we have $\left\langle f_{2}\left(x_{0}\right), u^{0}\right\rangle \leq 0$. But, the inequality $\left\langle f_{2}\left(x_{0}\right), u^{0}\right\rangle \geq 0$ is also valid, because $f_{2}\left(x_{0}\right) \in K_{2}$ and $u^{0} \in K_{2}^{*}$. Therefore we must have the equality $\left\langle f_{2}\left(x_{0}\right), u^{0}\right\rangle=0$. Consequently $u^{0}$ belongs to $L$. Now choose a number $\varepsilon>0$ for which

$$
A_{2}\left(t^{0}\right)-\varepsilon\left\|t^{0}\right\| y_{2}^{0}-\varepsilon u^{0} \in L^{*} .
$$

Since $A_{2}\left(t^{0}\right) \in$ int $L^{*}$, such a choice is possible. From (4.8) and $u^{0} \in L$ it follows that

$$
\left\langle A_{2}\left(t^{0}\right)-\varepsilon\left\|t^{0}\right\| y_{2}^{0}-\varepsilon u^{0}, u^{0}\right\rangle \geq 0
$$


and hence that

$$
\left\langle A_{2}\left(t^{0}\right)-\varepsilon\left\|t^{0}\right\| y_{2}^{0}, u^{0}\right\rangle \geq \varepsilon\left\langle u^{0}, u^{0}\right\rangle>0
$$

Because

$$
\lim _{k \rightarrow \infty}\left\langle A_{2}\left(t^{n_{k}}\right)-\varepsilon\left\|t^{n_{k}}\right\| y_{2}^{0}, u^{k}\right\rangle=\left\langle A_{2}\left(t^{0}\right)-\varepsilon\left\|t^{0}\right\| y_{2}^{0}, u^{0}\right\rangle
$$

it results from (4.9) that there is a number $k_{1} \in N$ such that

$$
\left\langle A_{2}\left(t^{n_{k}}\right)-\varepsilon\left\|t^{n_{k}}\right\| y_{2}^{0}, u^{k}\right\rangle>0 \text { for all } k_{1} \leq k \in N
$$

On the other hand, condition $\left(c_{2}\right)$ implies the existence of a number $r_{2} \in(0, r]$ such that

$$
\rho_{2}(t)+\varepsilon y_{2}^{0} \in K_{2} \quad \text { for all } t \in B_{+}^{m+1}\left(r_{2}\right) .
$$

To $r_{2}$ we can assign a number $k_{2} \in N$ such that $\left\|a_{n_{k}} t^{n_{k}}\right\| \leq r_{2}$ for all $k_{2} \leq k \in N$, because $\lim _{k \rightarrow \infty} a_{n_{k}} t^{n_{k}}=0$. In virtue of (4.11) it follows that

$$
\rho_{2}\left(a_{n_{k}} t^{n_{k}}\right)+\varepsilon y_{2}^{0} \in K_{2} \quad \text { for all } k_{2} \leq k \in N
$$

and hence that the points

$$
v^{k}=\left\|t^{n_{k}}\right\|\left(\rho_{2}\left(a_{n_{k}} t^{n_{k}}\right)+\varepsilon y_{2}^{0}\right) \text { for all } k_{2} \leq k \in N
$$

also lie in $K_{2}$. Therefore we have

$$
\left\langle v^{k}, u^{k}\right\rangle \geq 0 \text { for all } k_{2} \leq k \in N
$$

By addition (4.10) and (4.12) yield

$$
\left\langle A_{2}\left(t^{n_{k}}\right)+\left\|t^{n_{k}}\right\| \rho_{2}\left(a_{n_{k}} t^{n_{k}}\right), u^{k}\right\rangle>0 \text { for all } \max \left\{k_{1}, k_{2}\right\} \leq k \in N \text {. }
$$

This inequality implies

$$
\left\langle G\left(a_{n_{k}} t^{n_{k}}\right), u^{k}\right\rangle=\left\langle f_{2}\left(x_{0}\right), u^{k}\right\rangle+a_{n_{k}}\left\langle A_{2}\left(t^{n_{k}}\right)+\left\|t^{n_{k}}\right\| \rho_{2}\left(a_{n_{k}} t^{n_{k}}\right), u^{k}\right\rangle>0
$$

for all $\max \left\{k_{1}, k_{2}\right\} \leq k \in N$, which contradicts (4.7). In conclusion there must exist a number $p_{0} \in N$ such that (4.6) holds whenever $u \in U$. Therefore we have $\left\langle G\left(a_{n} t^{n}\right), u\right\rangle \geq$ 0 for all $p_{0} \leq n \in N$ and all $u \in K_{2}^{*}$. This means that

$$
G\left(a_{n} t^{n}\right) \in\left(K_{2}^{*}\right)^{*}=K_{2} \text { for all } p_{0} \leq n \in N
$$

From (a) and (4.13) it follows that

$$
f_{2}\left(\omega\left(a_{n} t^{n}\right)\right) \in K_{2}+G\left(a_{n} t^{n}\right) \subseteq K_{2}+K_{2} \subseteq K_{2} \quad \text { for all } p_{0} \leq n \in N .
$$

Together (4.5) and (4.14) express that

$$
\omega\left(a_{n} t^{n}\right) \in S \quad \text { for all } p_{0} \leq n \in N .
$$


After all we focus on $f_{1}$. Since $A_{1}\left(t^{0}\right) \in \operatorname{int} K_{1}$ we can select a number $\varepsilon>0$ such that

$$
A_{1}\left(t^{0}\right)-\varepsilon\left\|t^{0}\right\| y_{1}^{0} \in \text { int } K_{1} \text {. }
$$

Next, according to condition $\left(c_{1}\right)$, we can pick out a number $r_{1} \in(0, r]$ such that

$$
\rho_{1}(t)+\varepsilon y_{1}^{0} \in K_{1} \quad \text { for all } t \in B_{+}^{m+1}\left(r_{1}\right) \text {. }
$$

Now, taking into consideration that

$$
\lim _{n \rightarrow \infty}\left(A_{1}\left(t^{n}\right)-\varepsilon\left\|t^{n}\right\| y_{1}^{0}\right)=A_{1}\left(t^{0}\right)-\varepsilon\left\|t^{0}\right\| y_{1}^{0}
$$

and that

$$
\lim _{n \rightarrow \infty} a_{n} t^{n}=0
$$

we can choose a number $p_{1} \in N$ such that both properties

$$
A_{1}\left(t^{n}\right)-\varepsilon\left\|t^{n}\right\| y_{1}^{0} \in \operatorname{int} K_{1} \quad \text { and } \quad\left\|a_{n} t^{n}\right\| \leq r_{1}
$$

hold for all $p_{1} \leq n \in N$. In view of (4.18) and (4.16) we get

$$
\begin{aligned}
A_{1}\left(t^{n}\right)+\left\|t^{n}\right\| \rho_{1}\left(a_{n} t^{n}\right) & =A_{1}\left(t^{n}\right)-\varepsilon\left\|t^{n}\right\| y_{1}^{0}+\left\|t^{n}\right\|\left(\rho_{1}\left(a_{n} t^{n}\right)+\varepsilon y_{1}^{0}\right) \\
& \in \operatorname{int} K_{1}+K_{1} \\
& \subseteq \operatorname{int} K_{1}
\end{aligned}
$$

for all $p_{1} \leq n \in N$. By applying (a) it follows that

$$
\begin{aligned}
f_{1}\left(\omega\left(a_{n} t^{n}\right)\right) & \in f_{1}\left(x_{0}\right)+a_{n}\left(A_{1}\left(t^{n}\right)+\left\|t^{n}\right\| \rho_{1}\left(a_{n} t^{n}\right)\right)+K_{1} \\
& \subseteq f_{1}\left(x_{0}\right)+\operatorname{int} K_{1}+K_{1} \\
& \subseteq f_{1}\left(x_{0}\right)+\operatorname{int} K_{1}
\end{aligned}
$$

for all $p_{1} \leq n \in N$.

Since $x_{0}$ is a local solution to problem (WMOP), there exists a neighbourhood $V$ of $x_{0}$ such that

$$
\left(f_{1}\left(x_{0}\right)+\text { int } K_{1}\right) \cap f_{1}(S \cap V)=\emptyset .
$$

In virtue of (b) we can find a neighbourhood $W$ of the origin of the space $R^{m+1}$ such that $\omega(t) \in V$ for all $t \in W \cap B_{+}^{m+1}(r)$. According to (4.17), we can choose a number $p_{2} \in N$ such that

$$
a_{n} t^{n} \in W \text { for all } p_{2} \leq n \in N \text {. }
$$

Hence we have

$$
\omega\left(a_{n} t^{n}\right) \in V \text { for all } p_{2} \leq n \in N .
$$

Choosing any number $n \in N$ which satisfies $n \geq \max \left\{p_{0}, p_{1}, p_{2}\right\}$, we conclude from (4.19), (4.15) and (4.21) that the point $a_{n} t^{n}$ associated with this $n$ fulfils both inclusions

$$
f_{1}\left(\omega\left(a_{n} t^{n}\right)\right) \in f_{1}\left(x_{0}\right)+\operatorname{int} K_{1} \quad \text { and } \quad \omega\left(a_{n} t^{n}\right) \in S \cap V .
$$


This result contradicts (4.20). So it is proved that the origin of the space $R^{m}$ is not an interior point of $M$.

It is easily seen that $M$ is a convex set. By applying a well-known separation theorem (see [3: Satz 2.33]) it results that there is a vector

$$
\lambda=\left(\lambda_{1}, \lambda_{2}, \lambda_{3}\right) \in R^{m_{1}} \times R^{m_{2}} \times R^{m_{3}} \backslash\left\{\left(0_{1}, 0_{2}, 0_{3}\right)\right\}
$$

for which

$$
\sup \{\langle y, \lambda\rangle \mid y \in M\} \leq 0 .
$$

Fix any point $t=\left(t_{1}, \ldots, t_{m+1}\right) \in$ int $R_{+}^{m+1}$. Let $y_{1}$ be any point of $K_{1}$. Then we have

$$
\left(A_{1}(t)-n y_{1}, A_{2}(t), A_{3}(t)\right) \in M \quad \text { for all } n \in N .
$$

Therefore (4.22) implies

$$
\frac{1}{n}\langle A(t), \lambda\rangle \leq\left\langle y_{1}, \lambda_{1}\right\rangle \text { for all } n \in N .
$$

Letting $n \rightarrow \infty$ in this inequality, we get $\left\langle y_{1}, \lambda_{1}\right\rangle \geq 0$. Since $y_{1}$ was arbitrarily chosen in $K_{1}$, we have $\lambda_{1} \in K_{1}^{*}$. In the same way it can be proved that $\lambda_{2} \in\left(L^{*}\right)^{*}=L$ and that $\lambda_{3} \in K_{3}^{*}$. In conclusion, $\lambda$ belongs to $K_{1}^{*} \times L \times K_{3}^{*} \backslash\left\{\left(0_{1}, 0_{2}, 0_{3}\right)\right\}$.

Since $A(t) \in M$, we get from (4.22) that $\langle A(t), \lambda\rangle \leq 0$. This inequality can be rewritten as

$$
\left\langle t_{1}\left(-d^{1}\right)+\ldots+t_{m+1}\left(-d^{m+1}\right), \lambda\right\rangle \geq 0 .
$$

Since $t$ was arbitrarily chosen in int $R_{+}^{m+1}$, we have

$$
\lambda \in\left(\mathcal{K}\left(\left\{-d^{j} \mid j=1, \ldots, m+1\right\}\right)\right)^{*},
$$

and thus $\lambda \in\left\{-d^{j} \mid j=1, \ldots, m+1\right\}^{*}$. Consequently

$$
\left\{-d^{j} \mid j=1, \ldots, m+1\right\}^{*} \cap\left(K_{1}^{*} \times L \times K_{3}^{*}\right) \neq\left\{\left(0_{1}, 0_{2}, 0_{3}\right)\right\}
$$

holds.

But $d^{1}, \ldots, d^{m+1}$ were arbitrarily chosen in $D$. Therefore we can apply Proposition 2.1. So we obtain a vector

$$
\left(\lambda_{1}^{*}, \lambda_{2}^{*}, \lambda_{3}^{*}\right) \in K_{1}^{*} \times K_{2}^{*} \times K_{3}^{*} \backslash\left\{\left(0_{1}, 0_{2}, 0_{3}\right)\right\}
$$

satisfying (4.1) and (4.2)

By constructing suitable $K$-derived convex cones we can deduce from Theorem 4.1 various practical necessary optimality conditions. In order to avoid an extension of the present paper, such applications of Theorem 4.1 will be given in subsequent papers.

Acknowledgements. This paper has been finalized while the author was visiting the Institute of Optimization and Stochastics of the Martin-Luther-University HalleWittenberg in 1993. He would like to express his gratitude both to Deutsche Forschungsgemeinschaft for the financial support of this visit and Professor Alfred Göpfert for the fruitful discussions on the subject of the paper. 


\section{References}

[1] Breckner, W. W. and I. Kolumbán: A multiplier rule for constrained optimization problems containing state and control variables. Report. Cluj-Napoca: Babes-Bolyai Univ./Sem. Optim. Theory, Report No. 8 (1987), 1 - 22.

[2] Breckner, W. W. and I. Kolumbán: Multiplier rules for optimization problems with a finite number of constraints. Studia Univ. Babeş-Bolyai, Ser. Math. 33 (1988)1, 15 - 37.

[3] Elster, K.-H., Reinhardt, R., Schäuble, M. and G. Donath: Einführung in die nichilineare Optimierung (Math.-Naturwiss. Bibl.: Vol. 63). Leipzig: B. G. Teubner Verlagsges. 1977.

[4] Gittleman, A.: A general multiplier rule. J. Optim. Theory Appl. 7 (1971), 29 - 38.

[5] Goebel, M.: Zur Theorie optimaler Steuerprobleme im Banachraum und deren Anwendung auf elliptische Systeme. Diss. B. Freiberg (Germany): Bergakademie Freiberg 1978.

[6] Goebel, M.: On control problems for a quasilinear second order ordinary differential equation. Math. Nachr. 142 (1989), 277 - 286.

[7] Gōpfert, A., and R. Nehse: Vektoroptimierung : Theorie, Verfahren und Anwendungen (Math.-Naturwiss. Bibl.: Vol. 74). Leipzig: B. G. Teubner Verlagsges. 1990.

[8] Hestenes, M. R.: On variational theory and optimal control theory. SIAM J. Control 3 (1965), $23-48$.

[9] Hestenes, M. R.: Calculus of Variations and Optimal Control Theory. New York et al: John Wiley \& Sons Inc. 1966.

[10] Hestenes, M. R.: Optimization Theory. New York et al: John Wiley \& Sons Inc. 1975.

[11] Jahn, J.: Mathematical Vector Optimization in Partially Ordered Linear Spaces. Frankfurt/Main et al: Verlag Peter Lang 1986.

[12] Lin, J. G.: Maximal vectors and multi-objective optimization. J. Optim. Theory Appl. 18 (1976), $41-64$.

[13] Maruşciac, I.: On Fritz John type optimality criterion in multi-objective optimization. Math. - Rev. Anal. Numér. Théor. Approx., Ser. Anal. Numér. Théor. Approx. 11 (1982), $109-114$.

[14] Nieuwenhuis, J. W.: A general multiplier rule. J. Optim. Theory Appl. 31 (1980), 167 . 176.

[15] Podinovskii, V. V. and V. D. Nogin: Pareto-Optimal Solutions of Multicriteria Problems (in Russian). Moscow: Nauka 1982.

[16] Sawaragi, Y., Nakayama, H. and T. Tanino: Theory of Multiobjective Optimization. Orlando et al: Academic Press Inc. 1985.

[17] Singh, C.: Optimality conditions in multiobjective differentiable programming. J. Optim. Theory Appl. 53 (1987), 115 - 123.

[18] Tamura, K. and S. Miura: Necessary and sufficient conditions for local and global nondominated solutions in decision problems with multi-objectives. J. Optim. Theory Appl. 28 (1979), 501 - 523.

[19] Tuy, H.: On the convex approximation of nonlinear inequalities. Math. Operationsforsch. Statist. 5 (1974), 451 - 466.

[20] von Wolfersdorf, L.: On a method of Bittner for deriving maximum principles to optimal control processes. Math. Operationsforsch. Statist., Ser. Optimization 9 (1978), 197 . 205. 